\title{
Levetiracetam Monotherapy in Children with Epilepsy: A Systematic Review
}

\author{
Amerins Weijenberg ${ }^{1}$ - Oebele F. Brouwer ${ }^{1}$ - Petra M. C. Callenbach ${ }^{1}$
}

Published online: 27 May 2015

(c) The Author(s) 2015. This article is published with open access at Springerlink.com

\begin{abstract}
Background Levetiracetam, a second-generation antiepileptic drug (AED) with a good efficacy and safety profile, is licensed as monotherapy for adults and children older than 16 years with focal seizures with or without secondary generalization. However, it is increasingly being used off-label in younger children.

Objectives We critically reviewed the available evidence and discuss the present status of levetiracetam monotherapy in children 0-16 years old.

Data Sources We systematically searched the literature using PubMed, Web of Science and Embase up to August 2014 for articles on levetiracetam monotherapy in children. Keywords were levetiracetam, monotherapy and child*. The titles and abstracts of 532 articles were evaluated by AW, of which 480 were excluded. The full texts of the other 52 articles were assessed for relevance.

Results We covered one review, one opinion statement and 32 studies in this review, including four randomized controlled trials, ten open-label prospective studies, eight retrospective studies, and ten case reports. The formal evidence for levetiracetam monotherapy in children is minimal: it is potentially efficacious or effective as initial monotherapy in children with benign epilepsy with centrotemporal spikes. In all of the published studies, however, efficacy and tolerability of levetiracetam seemed to be good and comparable to other AEDs.
\end{abstract}

Petra M. C. Callenbach

p.m.c.tijink@umcg.nl

1 Department of Neurology, University Medical Centre Groningen, University of Groningen, $\mathrm{AB} 51$, P.O. Box 30 001, 9700 RB Groningen, The Netherlands
Conclusion The data of 32 studies on levetiracetam monotherapy in children were insufficient to confirm that levetiracetam is effective as initial monotherapy for different types of seizures and/or epilepsy syndromes. There is still an urgent need for well designed trials to justify the widespread use of levetiracetam monotherapy in children of all ages.

\section{Key Points}

Efficacy and tolerability of levetiracetam monotherapy in children, even in very young children, seems to be good.

Levetiracetam monotherapy in children remains offlabel because 32 studies have yielded insufficient formal evidence for its use.

\section{Introduction}

Levetiracetam is a second-generation anti-epileptic drug (AED) that has been on the market since 1999 in Europe as add-on therapy for adolescents from the age of 16 years with focal epilepsy.

Levetiracetam, (S)- $\alpha$-ethyl-2-oxo-1-pyrrolidine acetamide, is the $(S)$-enantiomer of the ethyl analogue of piracetam and shares its chemical structure with numerous nootropic drugs $[1,2]$. The mechanism of action differs structurally and functionally from other currently available AEDs as it binds to the synaptic vesicle protein $2 \mathrm{~A}$ (SV2A). The presence of SV2A in the presynaptic terminals suggests that its anti-epileptic function might be based 
on it affecting presynaptic events that regulate synaptic vesicle release [3]. Although its precise mechanism of action is not known, Nowack et al. [4] suggested that levetiracetam might modulate SV2 protein interactions. As a consequence, normal levels of SV2 and synaptotagmin (a SV2-binding protein) at the synapse are maintained, which may reduce seizures. It also plays a role in $\mathrm{Ca}^{2+}$ homeostasis by inhibiting ryanodine and IP3 receptor-dependent $\mathrm{Ca}^{2+}$ release from endoplasmic reticulum and by inhibiting $\mathrm{Ca}^{2+}$ entry through blocking of the L-type $\mathrm{Ca}^{2+}$ channels in hippocampal neurons [5].

Levetiracetam is almost completely absorbed after oral administration and its bioavailability is close to $100 \%$; it is unaffected by food [6]. Peak plasma concentrations occur in $1 \mathrm{~h}$ and steady state concentrations are achieved in 2 days if levetiracetam is taken twice daily. Pharmacokinetics is linear, dose proportional and time independent [6]. The distribution is close to the volume of intracellular and extracellular water and levetiracetam remains almost unattached to proteins [7]. Levetiracetam is minimally metabolized and, after $24 \mathrm{~h}, 27 \%$ is excreted as inactive metabolites [8]. The metabolism of levetiracetam does not involve the hepatic cytochrome P450 (CYP) system, nor does it inhibit or induce hepatic enzymes [6]. The major elimination route for levetiracetam is renal; $66 \%$ as an unchanged drug [9]. Dose adjustments are only recommended in patients with moderate to severe renal impairment or severe hepatic impairment with concomitant renal insufficiency. The body clearance of levetiracetam in children is $30-40 \%$ higher compared with adults and it is therefore recommended that children have a daily maintenance dose on a weight normalized level (20-60 mg/ $\mathrm{kg} /$ day) divided over two doses; this is equivalent to $130-140 \%$ of the usual daily adult maintenance dosage (1000-3000 mg/day) [10]. Levetiracetam has no clinically meaningful drug-drug interactions with other AEDs, or non-AEDs such as oral contraceptives, warfarin and digoxin. Thus, because of its unique chemical structure, specific mode of action and pharmacokinetic profile, levetiracetam has become one of the most widely used second-generation AEDs for both adults and children.

Levetiracetam was licensed as add-on therapy in children in 2005. Nowadays, levetiracetam is registered in Europe and the US as add-on therapy for focal onset seizures with or without secondary generalization in patients from 1 month of age, as add-on therapy for myoclonic seizures in patients from 12 years of age with juvenile myoclonic epilepsy, and as add-on therapy for primary generalized tonic-clonic seizures in patients with idiopathic generalized epilepsy (in Europe from 12 years of age; in the US from 6 years of age).

It was not until 2006 that it was licensed as monotherapy, but only in Europe, for adults and children from
16 years of age with focal onset seizures with or without secondary generalization. Off-label use of levetiracetam as monotherapy in younger children has increased considerably over the last decade due to its efficacy in both focal and generalized seizures, its good safety profile, favourable pharmacokinetic properties and its availability in an intravenous form for the acute setting [11-18].

Here, we review the available evidence for the use of levetiracetam monotherapy in children in the literature, including data from randomized controlled trials. We also discuss the present status of levetiracetam and make some recommendations for future research.

\section{Methods}

For this review, a literature search was performed by AW using PubMed (Medline), Web of Science and Embase (until August 2014) for papers on levetiracetam monotherapy in children $(<18$ years of age). There are no Cochrane Reviews on levetiracetam monotherapy. The following search terms were used: levetiracetam AND monotherapy AND child*. Only papers written in English, Dutch, French, or German were included. Articles were screened by AW and, in case of any dispute, discussed with PMCC. If a study included both children and adults, it was reviewed only if the results of efficacy were reported separately for children. We also searched the reference lists of these publications for more articles relevant to the topic. Abstracts of congress proceedings were not included. Data extraction from the articles was done independently by AW in Word and monitored by PMCC. We critically evaluated the study designs and whether there was any risk of bias in the individual studies.

\section{Overview of Published Articles}

The systematic literature search yielded 690 articles. After removing duplicates, the titles and abstracts of 532 articles were evaluated by AW; 480 were excluded (Fig. 1). The full texts of the other 52 articles were assessed for relevance and 34 articles were included in this systematic review: four randomized controlled trials (RCTs), ten open-label prospective studies, eight retrospective studies, ten case reports, a review, and an opinion statement [12, 19-51].

\subsection{Review, Opinion Statement and Case Reports}

One review and one opinion statement argued that levetiracetam monotherapy should be the drug of choice in patients with juvenile myoclonic epilepsy, based on evidence from trials, especially if valproic acid is contra- 


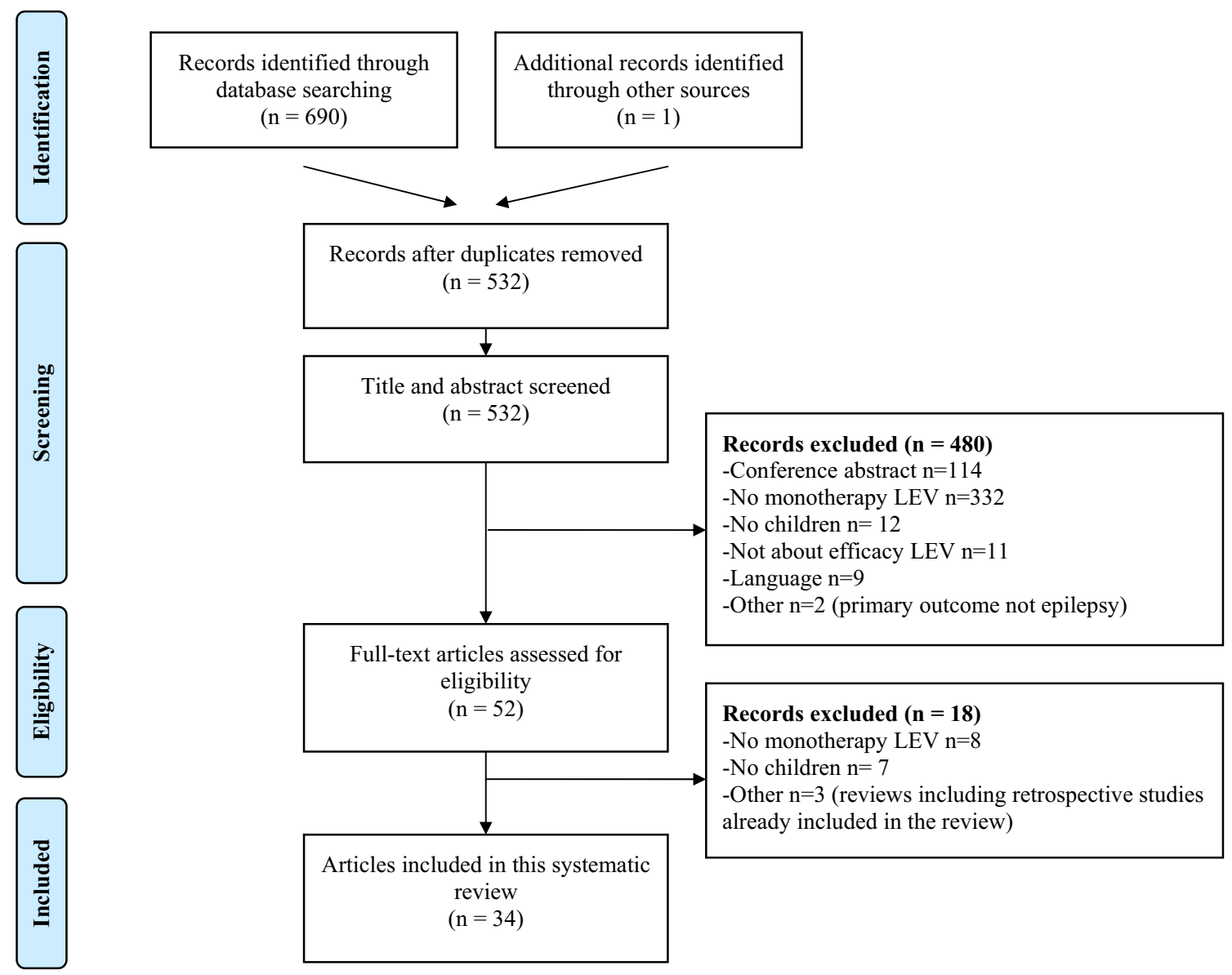

Fig. 1 PRISMA flow diagram. LEV levetiracetam

indicated; for example, in women of child-bearing age [50, 51].

Ten case reports have been published on the use of levetiracetam monotherapy in children, including neonates, with a wide variety of seizure types, epilepsy syndromes, dosages and ages (Table 1) [40-49]. Dosages of levetiracetam were given in $\mathrm{mg} / \mathrm{kg} / \mathrm{day}$ or $\mathrm{mg} / \mathrm{day}$ or not documented. All children became seizure-free, but the duration of follow-up was not given in three case reports (Table 1). The case reports suggested a high efficacy of treatment with levetiracetam monotherapy, and adverse events were infrequent or they were not reported.

\subsection{Retrospective Studies}

Eight retrospective studies on levetiracetam monotherapy in children have been published, the first in 2004 (Table 2) [32-39]. Most of them included patients with focal and/or generalized epilepsy. Levetiracetam dosage in these studies ranged from 10 to $108 \mathrm{mg} / \mathrm{kg} /$ day, but was mostly in the $20-40 \mathrm{mg} / \mathrm{kg} /$ day range. The mean duration of follow-up ranged from 3 to 27 months; four studies had a follow-up of more than 1 year $[34,35,37,38]$. Three studies compared efficacy and tolerability of levetiracetam with carbamazepine [35], with oxcarbazepine or valproic acid [36], and with valproic acid [38].

In all but one of the eight studies, levetiracetam efficacy was considered to be good, and seizure freedom was achieved in more than $60 \%$ of patients in most studies, including those on children who had been using another AED prior to levetiracetam monotherapy (Table 2). Tolerability was good in all studies, with behavioural and cognitive changes being the most common adverse events; the discontinuation rate due to adverse events was low $(0-12 \%)$. In one study, the retention rate was not significantly different between the two groups (levetiracetam vs oxcarbazepine and levetiracetam vs valproic acid), although levetiracetam monotherapy failed more often due to lack of efficacy in both groups [36].

\subsection{Prospective Open-Label Studies}

Lagae et al. [12] were the first to report a prospective trial on levetiracetam monotherapy in children. Since then, nine more open-label prospective studies have been published that included children, and sometimes even neonates, with 


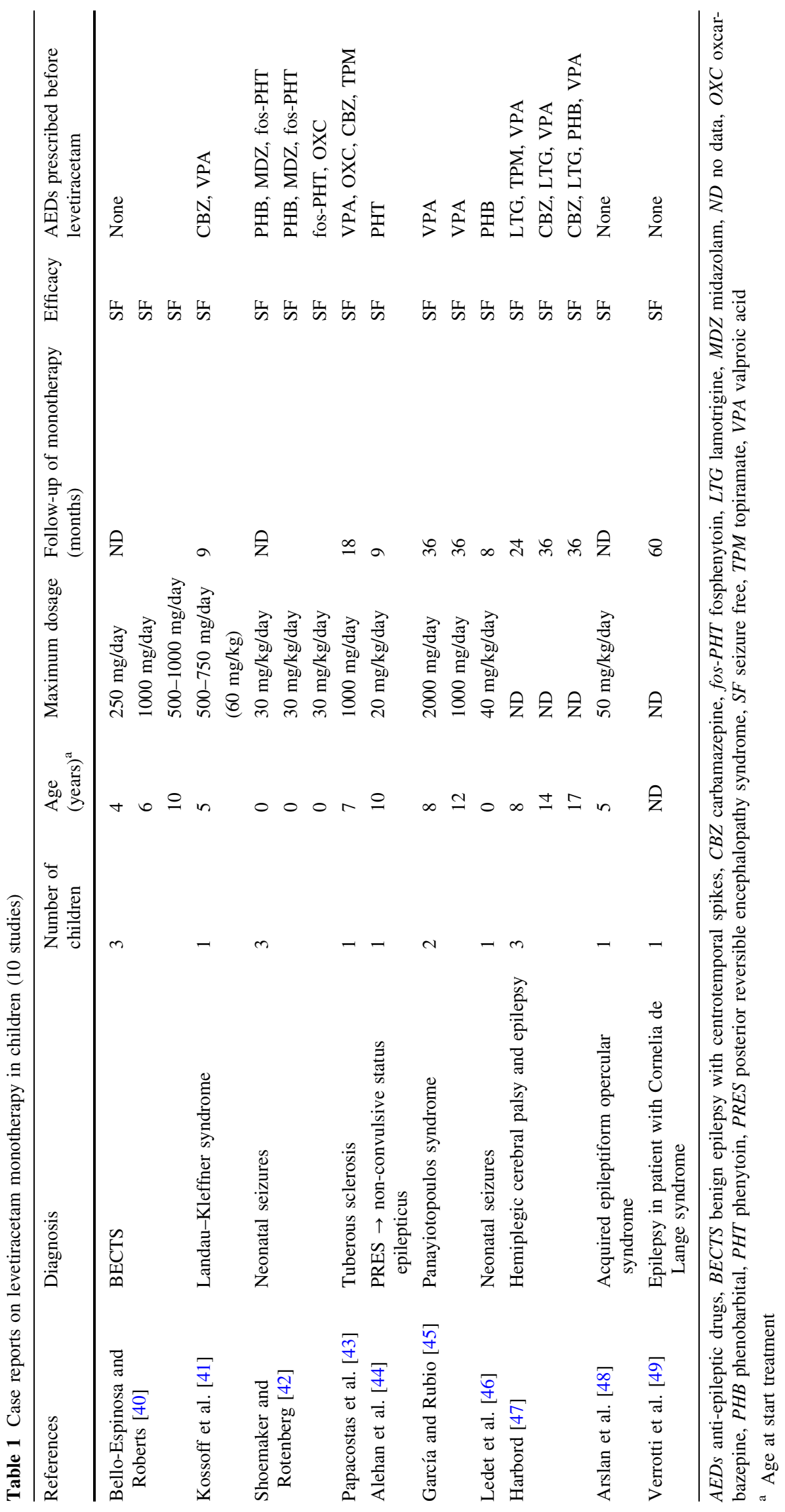




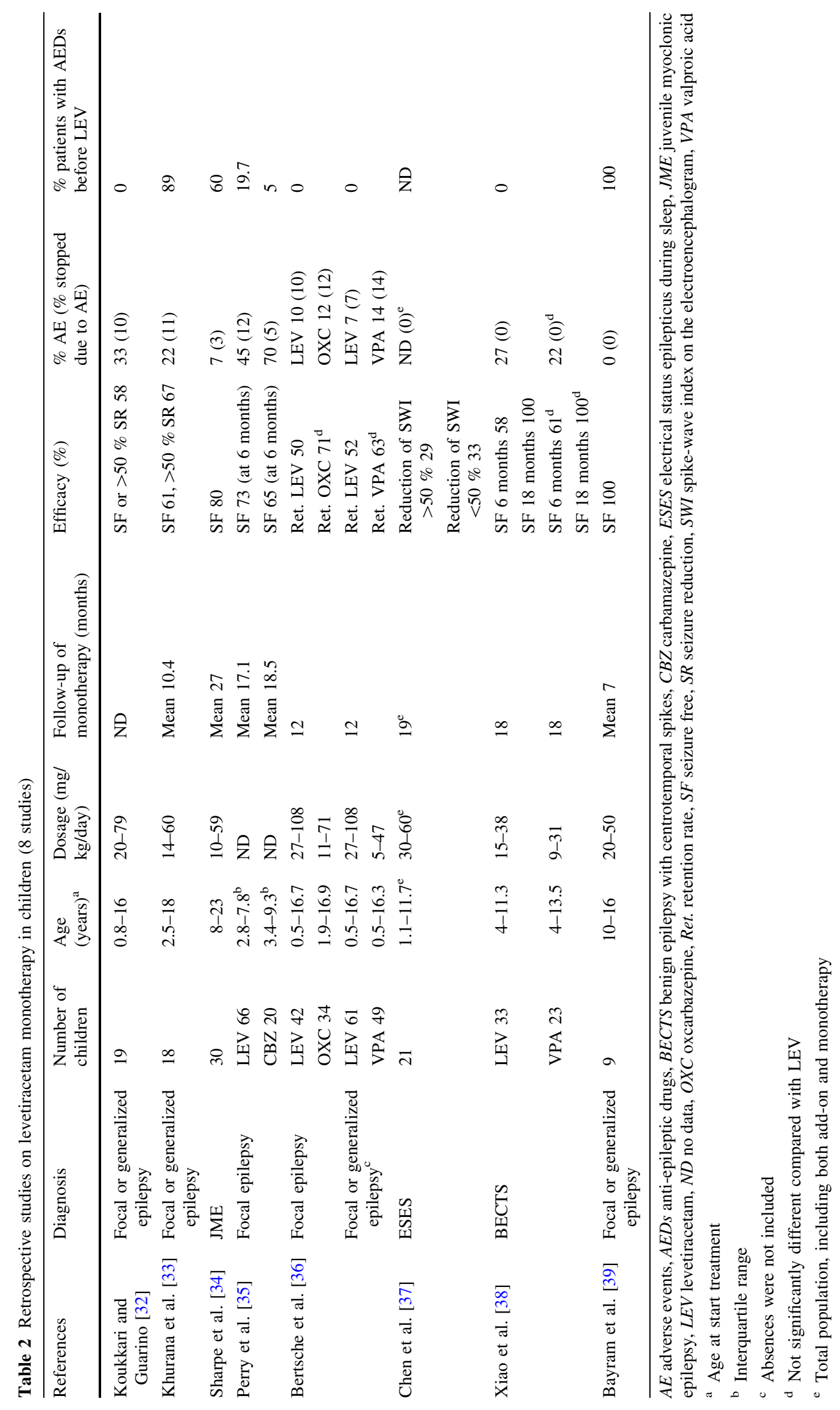


different but overall relatively benign seizure types and/or epilepsy syndromes (Table 3) [23-31]. In three studies, levetiracetam was given in $\mathrm{mg} / \mathrm{day}$, without considering body weight, with dosages ranging from 1000 to $3000 \mathrm{mg} /$ day [23, 24, 27]. In seven studies, dosages were based on bodyweight, starting with $10 \mathrm{mg} / \mathrm{kg} /$ day, with increasing dosage until seizure freedom was reached, with a maximum of $70 \mathrm{mg} / \mathrm{kg} /$ day (Table 3) [12, 25, 26, 28-31]. In a pilot study by Kossoff et al. [26], children were switched from carbamazepine or oxcarbazepine monotherapy to levetiracetam monotherapy. The baseline AED was tapered off over 2 weeks. Verrotti et al. [24] reported on 21 children who received levetiracetam monotherapy: 12 of them were converted from monotherapy with valproic acid, carbamazepine, oxcarbazepine, or lamotrigine to levetiracetam monotherapy. However, follow-up was more than 1 year in only half of these trials; this is regarded as the minimum duration to draw any conclusions about long-term efficacy, adverse events and tolerability of AED treatment [52, 53].

In most studies, efficacy of levetiracetam monotherapy was reported to be good, with a high percentage of children becoming seizure free $(20-100 \%)$ or having more than $50 \%$ seizure reduction $(62-100 \%)$. In one study, the response was significantly better in the children who were AED-naive before initiating treatment with levetiracetam [24]. Some studies used extra outcome parameters other than efficacy, such as quality of life [12], electro-encephalography (EEG) findings and language function [26]. Lagae et al. [12] studied ten children and observed increased alertness in three and a positive effect on behaviour in one. Furthermore, median overall quality of life was higher in children on levetiracetam monotherapy than in children with add-on levetiracetam [12]. Kossoff et al. [26] evaluated EEG findings and language function in six children with benign epilepsy with centrotemporal spikes (BECTS), and they also looked for additional evidence of impaired auditory comprehension and verbal memory. After 6 months of treatment, the parents of all children reported subjective improvements, which were confirmed in most children by objective testing. The EEG had normalized in only three children.

Six trials reported the occurrence of adverse events, most commonly irritability and somnolence [12, 24, 26, 28, $29,31]$. In three, all adverse events were transient [24, 28, 29] and in two, none of the children discontinued levetiracetam because of adverse events [12, 26]. In a large study of 37 children on levetiracetam monotherapy and 83 children on levetiracetam add-on treatment, a relatively high percentage reported adverse events $(47.5 \%)$ and four of them even had to discontinue levetiracetam [31]. Results for the group treated with monotherapy were, however, not given separately.

\subsection{Randomized Controlled Trials}

Four RCTs have been published: two open-label parallel group trials and two double-blind trials (Table 4) [19-22]. Most trials only included children with a well described epilepsy syndrome such as BECTS [19, 22] or absence epilepsy (childhood absence epilepsy [CAE], juvenile absence epilepsy [JAE]) [20]. The age at enrolment varied between 3 and 17 years. The maximum dosage of levetiracetam was $2000 \mathrm{mg} /$ day in 12- to 17-year-old children [21] or $30 \mathrm{mg} / \mathrm{kg} /$ day $[19,20,22]$. One trial was placebocontrolled [20] and, for obvious ethical reasons [54], the duration of the double-blind period was only 2 weeks, which is much shorter than the duration of the other trials (24-78 weeks).

The equivalence open-label trial of Coppola et al. [19] compared levetiracetam with oxcarbazepine in children with BECTS and they observed no significant difference in the percentage being seizure free at 18 months (Table 4). In another trial, in children with absence epilepsy, Fattore et al. [20] showed no significant difference in seizure freedom between levetiracetam and placebo. After the 2-week double-blind period, the trial continued as an openlabel trial and almost all children receiving the placebo were switched to levetiracetam. During long-term followup, $32 \%(12 / 38)$ of the children initially on levetiracetam continued with levetiracetam and were seizure-free for at least 267 days; $63 \%$ (24/38) discontinued levetiracetam because of inefficacy at a later stage. After 1 year, 17 children (29\%) were still seizure-free on levetiracetam (initially on levetiracetam or placebo therapy). Rosenow et al. [21] included patients aged $\geq 12$ years with newly diagnosed focal or generalized epilepsy. If patients were already using an AED, this was tapered off during the first 3 weeks of the study period. A post-hoc subgroup analysis was performed for 33 patients aged 12-17 years. Seizure freedom after 6 weeks of treatment was compared between levetiracetam and lamotrigine, although patients on lamotrigine were still in their titration period and the dosage of lamotrigine also increased after this time-point. Efficacy and tolerability of levetiracetam and lamotrigine did not differ significantly for the group aged 12-17 years. Quality-of-life scores (QOLIE-10) at the beginning and end of treatment (26 weeks) were similar in both treatment groups; a subgroup analysis for children aged 12-17 was not presented. In the non-inferiority trial of Borggraefe et al. [22], levetiracetam was compared with sulthiame in children with BECTS. Their primary endpoint was treatment failure, defined by seizure recurrence during the observation period. This was not significantly different between treatments (Table 4). However, the retention rate was significantly higher in the sulthiame group than in the levetiracetam group $(p=0.03)$. 


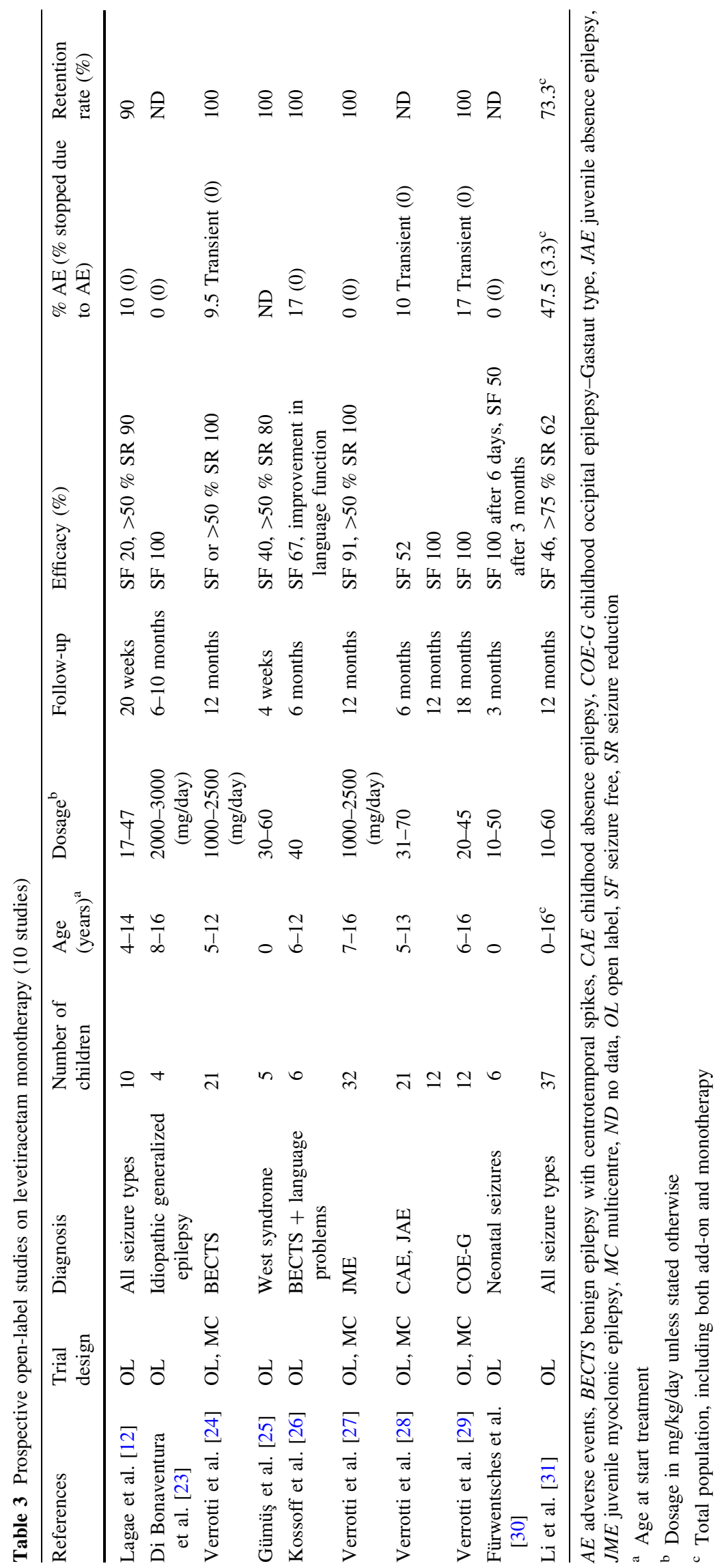




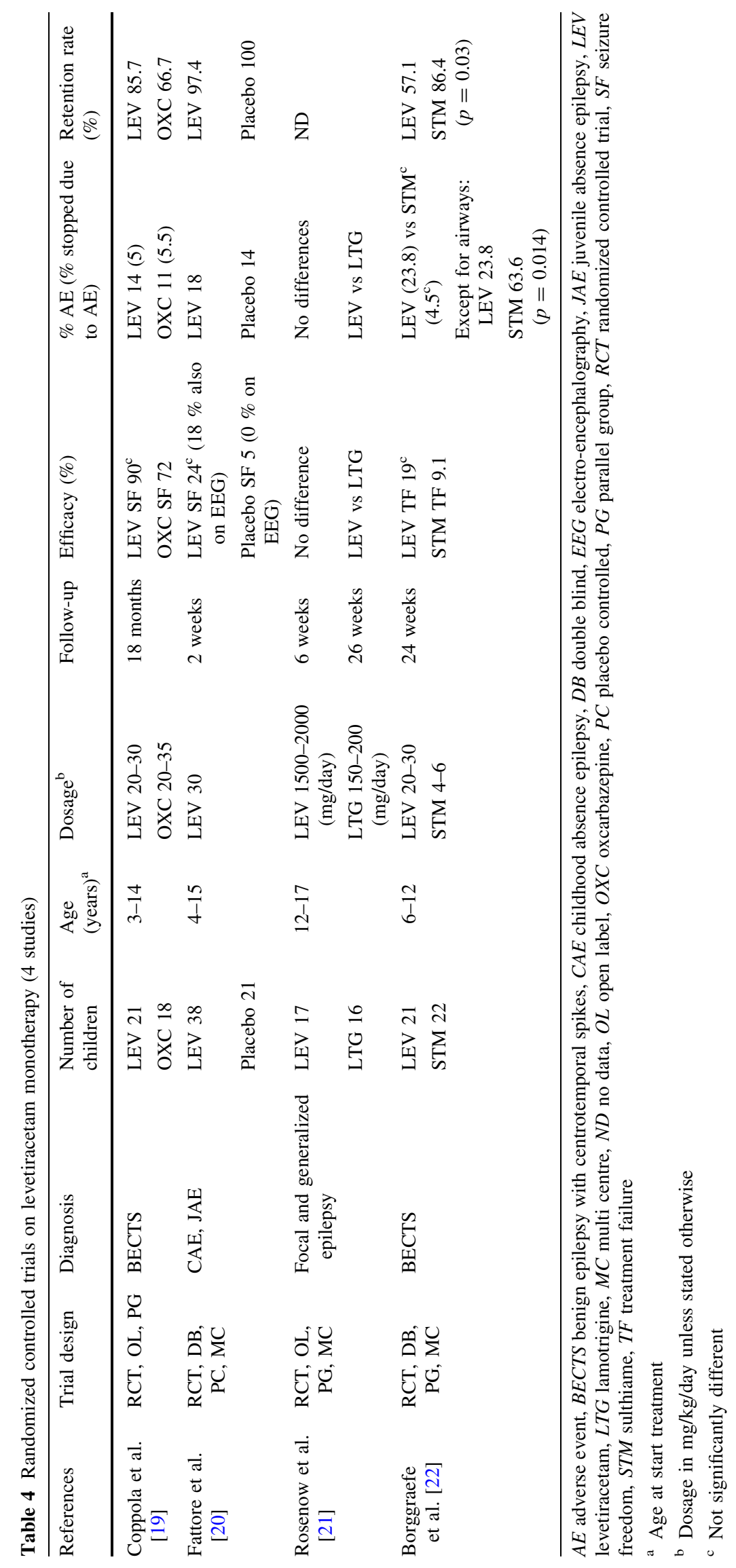


The most commonly reported adverse events were somnolence and irritability or behavioural problems. Significant differences with respect to adverse events were not observed between the treatments reported. However, none of the trials used a standardized questionnaire to investigate the occurrence of adverse events.

\section{Discussion}

In this review, 32 studies on levetiracetam monotherapy in children are described. In all of them, efficacy and tolerability of levetiracetam monotherapy seems to be good and comparable or even favourable to other AEDs. Nonetheless, we must recognize that it has only been licensed for monotherapy in children older than 16 years in Europe.

\subsection{Current Evidence for Efficacy}

The most commonly used primary endpoint for efficacy was seizure freedom and/or percentage of seizure reduction (30 of 32 studies). The case reports suggested a very high efficacy of treatment with levetiracetam monotherapy. Publication bias may, however, have led to an unrealistic positive view of its efficacy. The percentage of children becoming seizure-free in both retrospective and prospective studies was $61-100 \%$. Only $20-46 \%$ of cases became seizure-free in three prospective open-label studies that included children with all seizure types or even West syndrome [12, 25, 31]. Efficacy of levetiracetam does not seem to be related to age at enrolment, dosage or seizure type and/or epilepsy syndrome (Tables 1, 2, 3, 4). Since most studies included children with overall relatively benign seizure types and/or syndromes, its efficacy might be overestimated. In summary, the efficacy of levetiracetam monotherapy in children seems good and comparable to other AEDs, but the level of evidence is limited and not available for all seizure types and/or epilepsy syndromes.

\subsection{Current Knowledge on Tolerability}

The range of children with reported adverse events varied between 0 and $47.5 \%$. The percentage of children who had to stop levetiracetam treatment due to adverse events was $0-12 \%$ in most studies, although in the trial of Borggraefe et al. [22], $23.8 \%$ of the children discontinued levetiracetam because of adverse events. The most frequently reported adverse events were behavioural and/or cognitive changes (i.e. irritability, mood disturbances or somnolence), but these complaints were mostly transient. This is in line with the most commonly reported adverse events for levetiracetam [55]. In children with pre-existing behavioural problems, the problems could be exacerbated during levetiracetam therapy [56]. In some, but not all, of the prospective studies, pre-existing behavioural and/or cognitive problems were exclusion criteria [24, 26-29]. In two RCTs, children with a mental deficit or intellectual disability were excluded $[19,20]$. The other two RCTs did not mention any exclusion criteria for behavioural and/or cognitive problems [21, 22]. Because children with epilepsy may develop cognitive and/or behavioural problems due to both the epilepsy itself and the treatment with AEDs, it is very important to evaluate the exact role of treatment on these problems as well $[54,57]$. According to Cross et al. [58], effective management requires treatment within the context of the overall health status and quality of life of the treated child. Two trials investigated quality of life, and this was unchanged or positively influenced by the use of levetiracetam monotherapy [12, 21].

In summary, the tolerability of levetiracetam seems to be good, with only a few adverse events that are mostly transient, even in very young children and in dosages up to $70 \mathrm{mg} / \mathrm{kg} /$ day.

\subsection{Evaluation of Study Design and Methodology}

According to the International League Against Epilepsy (ILAE), the best evidence for the use of levetiracetam monotherapy in children up to March 31, 2012 reached level D for children with BECTS, based on the study by Coppola et al. [19, 53]. Level D means there is one class III double-blind or open-label study, or one or more class IV clinical studies or data from expert committee reports or opinions from experienced clinicians [53]. Since 2012, two more RCTs on levetiracetam monotherapy have been published, both with a class III rating [21, 22]. Because of the inconclusive trial results of Borggraefe et al. [22], the level of evidence for levetiracetam monotherapy in BECTS did not reach level $\mathrm{C}$, while the trial results of Rosenow et al. [21] could not contribute to the level of evidence for levetiracetam monotherapy in focal and generalized epilepsy because of their study design.

Prospective studies may also contribute to the level of evidence. However, eight of the ten prospective studies did not perform a formal statistical evaluation, and only one of the other two studies found a significant decrease in seizure frequency in a subgroup analysis [12, 23-31]. Although these ten studies did not contribute to the level of evidence for levetiracetam monotherapy in children, the percentage of children becoming seizure-free after the start of levetiracetam is promising, although there may well be some publication bias.

The reasons for the small number of prospective trials performed in children are pharmaceutical companies' lack of interest in such a small market when the patent has expired, and the difficulties in recruiting patients, partially 
due to ethical and legal aspects [59]. Moreover, in the past, separate drug trials in children were not required. As a consequence, levetiracetam is often prescribed off-label for children based on the results of trials in adults. Children, however, have a different developmental physiology, disease pathophysiology, pharmacokinetics and/or pharmacodynamics, resulting in treatment responses that are unpredictably different from those in adults $[60,61]$. There is therefore an urgent need for clinical trials in children, because $<50 \%$ of medicines used in children have been properly studied in this age group [62]. For example, antiepileptic drugs that have been registered as add-on therapy in children and/or as monotherapy in adults should also be studied as monotherapy in children; this is already obligatory for drugs now being developed.

The ILAE has described an ideal design for clinical trials in children [53]. This includes a randomized double-blind design, with adequate sample size calculations leading to a large enough study population to show non-inferiority with a $\leq 20 \%$ relative difference between treatment arms, based on $80 \%$ power in a non-inferiority analysis versus an acceptable comparator; with retention rate or seizure freedom as the primary endpoint after a minimum of 48 weeks of treatment, and an appropriate statistical analysis. Of the four RCTs on levetiracetam in children, only two were doubleblind studies [19, 22]. Remarkably, these two studies compared levetiracetam with oxcarbazepine or sulthiame in children with BECTS, whereas the level of evidence for the efficacy of both oxcarbazepine and sulthiame is low [19, 22, 53]. Carbamazepine and valproic acid would have been a more obvious choice for comparison of efficacy [53]. Furthermore, the follow-up duration in the Borggraefe et al. [22] study was only 24 weeks.

In our opinion, one of the best ways to measure efficacy, side effects and tolerability is by using retention rate, because this endpoint combines all these parameters [52]. Retention rates in the prospective open-label studies and RCTs ranged from 57.1 to $100 \%$ (Tables 3, 4).

One limitation of our review is that we did not include conference papers and that the literature search and screening of articles was done by only one person. Another is that we did not perform a meta-analysis. This was not possible due to the heterogeneity of the population with varying epilepsy syndromes and seizure types, the variation in study designs and the different AEDs used for comparison.

\section{Conclusion and Recommendations}

The formal evidence for the use of levetiracetam monotherapy in children remains quite scarce: it is potentially efficacious or effective as initial monotherapy in children with BECTS. Because of the study designs and the limited number of trials, there is insufficient data available to confirm that levetiracetam is effective as initial monotherapy in children for different types of seizures and/or epilepsy syndromes, other than BECTS.

More importantly, however, in the studies we evaluated, the efficacy of levetiracetam monotherapy in children seems at least equally comparable to other AEDs. The spectrum of reported adverse events is favourable, and levetiracetam does not have a negative impact on cognition [63]. Together with its availability in an intravenous form, unique chemical structure, novel mode of action and pharmacokinetic profile, levetiracetam may become one of the most important AEDs in treating children with epilepsy.

To formally justify the widespread use of levetiracetam monotherapy in children of all ages, we need more well conducted, double-blind RCTs to evaluate the efficacy, side effects and tolerability of levetiracetam monotherapy in children.

Acknowledgments We thank Ms. J. Senior for editing the final version of the text.

Compliance with ethical standards $\mathrm{PMCC}$ had an unrestricted research Grant from UCB Pharma BV, The Netherlands. OFB had an unrestricted educational Grant and payment for lectures from UCB Pharma BV, The Netherlands. UCB Pharma BV had no role in the study design, data collection and analysis, decision to publish, or preparation of the manuscript. AW has no conflicts of interest.

We confirm that this article does not contain any studies with human participants performed by any of the authors.

Open Access This article is distributed under the terms of the Creative Commons Attribution-NonCommercial 4.0 International License (http://creativecommons.org/licenses/by-nc/4.0/), which permits any noncommercial use, distribution, and reproduction in any medium, provided you give appropriate credit to the original author(s) and the source, provide a link to the Creative Commons license, and indicate if changes were made.

\section{References}

1. Shorvon S. Pyrrolidone derivatives. Lancet. 2001;358:1885-92.

2. Patsalos PN. Clinical pharmacokinetics of levetiracetam. Clin Pharmacokinet. 2004;43:707-24.

3. Lynch BA, Lambeng N, Nocka K, et al. The synaptic vesicle protein SV2A is the binding site for the antiepileptic drug levetiracetam. Proc Natl Acad Sci USA. 2004;101:9861-6.

4. Nowack A, Malarkey EB, Yao J, et al. Levetiracetam reverses synaptic deficits produced by overexpression of SV2A. PLoS One. 2011;6:e29560.

5. Deshpande LS, Delorenzo RJ. Mechanisms of levetiracetam in the control of status epilepticus and epilepsy. Front Neurol. 2014;5:11.

6. UCB. Keppra (levetiracetam 250, 500, 750 and, $1000 \mathrm{mg}$ tablets and $100 \mathrm{mg} / \mathrm{ml}$ for solution of infusion): summary of product characteristics. London: European Medicine Agency; 2010.

7. Doheny HC, Ratnaraj N, Whittington MA, et al. Blood and cerebrospinal fluid pharmacokinetics of the novel anticonvulsant 
levetiracetam (ucb L059) in the rat. Epilepsy Res. 1999;34:161-8.

8. Perucca E, Bialer M. The clinical pharmacokinetics of the newer antiepileptic drugs. Focus on topiramate, zonisamide and tiagabine. Clin Pharmacokinet. 1996;31:29-46.

9. Walker MC, Patsalos PN. Clinical pharmacokinetics of new antiepileptic drugs. Pharmacol Ther. 1995;67:351-84.

10. Pellock JM, Glauser TA, Bebin EM, et al. Pharmacokinetic study of levetiracetam in children. Epilepsia. 2001;42:1574-9.

11. Tan MJ, Appleton RE. Efficacy and tolerability of levetiracetam in children aged 10 years and younger: a clinical experience. Seizure. 2004;13:142-5.

12. Lagae L, Buyse G, Ceulemans B. Clinical experience with levetiracetam in childhood epilepsy: an add-on and mono-therapy trial. Seizure. 2005;14:66-71.

13. Glauser TA, Ayala R, Elterman RD, et al. Double-blind placebocontrolled trial of adjunctive levetiracetam in pediatric partial seizures. Neurology. 2006;66:1654-60.

14. Callenbach PM, Arts WF, ten Houten R, et al. Add-on levetiracetam in children and adolescents with refractory epilepsy: results of an open-label multi-centre study. Eur J Paediatr Neurol. 2008; 12:321-7.

15. Chung AM, Eiland LS. Use of second-generation antiepileptic drugs in the pediatric population. Paediatr Drugs. 2008;10:217-54.

16. van de Vrie-Hoekstra NW, de Vries TW, van den Berg PB, et al. Antiepileptic drug utilization in children from 1997-2005-a study from The Netherlands. Eur J Clin Pharmacol. 2008;64:1013-20.

17. Legros B, Boon P, De Jonghe P, et al. Opinion of Belgian neurologists on antiepileptic drug treatment in 2006: Belgian study on epilepsy treatment (BESET-2). Acta Neurol Scand. 2009;120:402-10.

18. Hsia Y, Neubert A, Sturkenboom MC, et al. Comparison of antiepileptic drug prescribing in children in three European countries. Epilepsia. 2010;51:789-96.

19. Coppola G, Franzoni E, Verrotti A, et al. Levetiracetam or oxcarbazepine as monotherapy in newly diagnosed benign epilepsy of childhood with centrotemporal spikes (BECTS): an open-label, parallel group trial. Brain Dev. 2007;29:281-4.

20. Fattore C, Boniver C, Capovilla G, et al. A multicenter, randomized, placebo-controlled trial of levetiracetam in children and adolescents with newly diagnosed absence epilepsy. Epilepsia. 2011;52:802-9.

21. Rosenow F, Schade-Brittinger C, Burchardi N, et al. The LaLiMo Trial: lamotrigine compared with levetiracetam in the initial 26 weeks of monotherapy for focal and generalised epilepsy-an open-label, prospective, randomised controlled multicenter study. J Neurol Neurosurg Psychiatry. 2012;83:1093-8.

22. Borggraefe I, Bonfert M, Bast T, et al. Levetiracetam vs. sulthiame in benign epilepsy with centrotemporal spikes in childhood: a double-blinded, randomized, controlled trial (German HEAD Study). Eur J Paediatr Neurol. 2013;17:507-14.

23. Di Bonaventura C, Fattouch J, Mari F, et al. Clinical experience with levetiracetam in idiopathic generalized epilepsy according to different syndrome subtypes. Epileptic Disord. 2005;7:231-5.

24. Verrotti A, Coppola G, Manco R, et al. Levetiracetam monotherapy for children and adolescents with benign rolandic seizures. Seizure. 2007;16:271-5.

25. Gümüş H, Kumandaş S, Per H. Levetiracetam monotherapy in newly diagnosed cryptogenic West syndrome. Pediatr Neurol. 2007;37:350-3

26. Kossoff EH, Los JG, Boatman DF. A pilot study transitioning children onto levetiracetam monotherapy to improve language dysfunction associated with benign rolandic epilepsy. Epilepsy Behav. 2007;11:514-7.
27. Verrotti A, Cerminara C, Coppola G, et al. Levetiracetam in juvenile myoclonic epilepsy: long-term efficacy in newly diagnosed adolescents. Dev Med Child Neurol. 2008;50:29-32.

28. Verrotti A, Cerminara C, Domizio S, et al. Levetiracetam in absence epilepsy. Dev Med Child Neurol. 2008;50:850-3.

29. Verrotti A, Parisi P, Loiacono G, et al. Levetiracetam monotherapy for childhood occipital epilepsy of gastaut. Acta Neurol Scand. 2009;120:342-6.

30. Fürwentsches A, Bussmann C, Ramantani G, et al. Levetiracetam in the treatment of neonatal seizures: a pilot study. Seizure. 2010;19:185-9.

31. Li J, Xiao N, Chen S. Efficacy and tolerability of levetiracetam in children with epilepsy. Brain Dev. 2011;33:145-51.

32. Koukkari MW, Guarino EJ. Retrospective study of the use of levetiracetam in childhood seizure disorders. J Child Neurol. 2004;19:944-7.

33. Khurana DS, Kothare SV, Valencia I, et al. Levetiracetam monotherapy in children with epilepsy. Pediatr Neurol. 2007;36:227-30.

34. Sharpe DV, Patel AD, Abou-Khalil B, et al. Levetiracetam monotherapy in juvenile myoclonic epilepsy. Seizure. 2008; 17:64-8.

35. Perry S, Holt P, Benatar M. Levetiracetam versus carbamazepine monotherapy for partial epilepsy in children less than 16 years of age. J Child Neurol. 2008;23:515-9.

36. Bertsche A, Neininger MP, Dahse AJ, et al. Initial anticonvulsant monotherapy in routine care of children and adolescents: levetiracetam fails more frequently than valproate and oxcarbazepine due to a lack of effectiveness. Eur J Pediatr. 2014;173:87-92.

37. Chen XQ, Zhang WN, Yang ZX, et al. Efficacy of levetiracetam in electrical status epilepticus during sleep of children: a multicenter experience. Pediatr Neurol. 2014;50:243-9.

38. Xiao F, An D, Deng H, et al. Evaluation of levetiracetam and valproic acid as low-dose monotherapies for children with typical benign childhood epilepsy with centrotemporal spikes (BECTS). Seizure. 2014;23:756-61.

39. Bayram E, Topcu Y, Tufekci O, et al. The efficacy and safety of levetiracetam in paediatric patients treated with chemotherapeutic agents for haematologic disorders. J Paediatr Child Health. 2014;50:553-6.

40. Bello-Espinosa LE, Roberts SL. Levetiracetam for benign epilepsy of childhood with centrotemporal spikes-three cases. Seizure. 2003;12:157-9.

41. Kossoff EH, Boatman D, Freeman JM. Landau-Kleffner syndrome responsive to levetiracetam. Epilepsy Behav. 2003;4:571-5.

42. Shoemaker MT, Rotenberg JS. Levetiracetam for the treatment of neonatal seizures. J Child Neurol. 2007;22:95-8.

43. Papacostas SS, Papathanasiou ES, Myrianthopoulou P, et al. Tuberous sclerosis successfully treated with levetiracetam monotherapy: 18 months of follow-up. Pharm World Sci. 2007;29:350-2.

44. Alehan F, Ozcay F, Haberal M. The use of levetiracetam in a child with nonconvulsive status epilepticus. J Child Neurol. 2008;23:331-3.

45. García C, Rubio G. Efficacy and safety of levetiracetam in the treatment of Panayiotopoulos syndrome. Epilepsy Res. 2009;85:318-20.

46. Ledet DS, Wheless JW, Rubnitz JE, et al. Levetiracetam as monotherapy for seizures in a neonate with acute lymphoblastic leukemia. Eur J Paediatr Neurol. 2010;14:78-9.

47. Harbord MG. Levetiracetam in children and adolescents with epilepsy and hemiplegic cerebral palsy. J Paediatr Child Health. 2011;47:302-4.

48. Arslan M, Yis U, Vurucu S, et al. Acquired epileptiform opercular syndrome: F-18 fluorodeoxyglucose positron emission 
tomography (FDG-PET) findings and efficacy of levetiracetam therapy. Epilepsy Behav. 2012;25:50-3.

49. Verrotti A, Agostinelli S, Prezioso G, et al. Epilepsy in patients with Cornelia de Lange syndrome: a clinical series. Seizure. 2013;22:356-9.

50. Mantoan L, Walker M. Treatment options in juvenile myoclonic epilepsy. Curr Treat Options Neurol. 2011;13:355-70.

51. Montouris G, Abou-Khalil B. The first line of therapy in a girl with juvenile myoclonic epilepsy: should it be valproate or a new agent? Epilepsia. 2009;50(Suppl 8):16-20.

52. Weijenberg A, Offringa M, Brouwer OF, et al. RCTs with new antiepileptic drugs in children: a systematic review of monotherapy studies and their methodology. Epilepsy Res. 2010;91:1-9.

53. Glauser T, Ben-Menachem E, Bourgeois B, et al. Updated ILAE evidence review of antiepileptic drug efficacy and effectiveness as initial monotherapy for epileptic seizures and syndromes. Epilepsia. 2013;54:551-63.

54. Seidenberg M, Pulsipher DT, Hermann B. Cognitive progression in epilepsy. Neuropsychol Rev. 2007;17:445-54.

55. Lyseng-Williamson KA. Levetiracetam: a review of its use in epilepsy. Drugs. 2011;71:489-514.
56. Verrotti A, D'Adamo E, Parisi P, et al. Levetiracetam in childhood epilepsy. Paediatr Drugs. 2010;12:177-86.

57. Plioplys S, Dunn DW, Caplan R. 10-year research update review: psychiatric problems in children with epilepsy. J Am Acad Child Adolesc Psychiatry. 2007;46:1389-402.

58. Cross JH, Kluger G, Lagae L. Advancing the management of childhood epilepsies. Eur J Paediatr Neurol. 2013;17:334-47.

59. Amann JP, Glauser T, Chiron C. Developing antiepileptic drugs in children: balancing protection and access. Handb Clin Neurol. 2013;111:741-6.

60. Caldwell PH, Murphy SB, Butow PN, et al. Clinical trials in children. Lancet. 2004;364:803-11.

61. Klassen TP, Hartling L, Craig JC, et al. Children are not just small adults: the urgent need for high-quality trial evidence in children. PLoS Med. 2008;5:e172.

62. Conroy S, Choonara I, Impicciatore P, et al. Survey of unlicensed and off label drug use in paediatric wards in European countries. European Network for Drug Investigation in Children. BMJ. 2000;320:79-82.

63. Ijff DM, Aldenkamp AP. Cognitive side-effects of antiepileptic drugs in children. Handb Clin Neurol. 2013;111:707-18. 